\title{
Rechtsprechung
}

\section{EuGH: Kein Urheberrechtsschutz für Geschmack eines Lebensmittels}

\author{
EuGH Urteil vom 13.11.2018 - C.310/17
}

\section{ECLI:EU:C:2018:899}

- In der Rechtssache C-310/17

betreffend ein Vorabentscheidungsersuchen nach Art. 267 AEUV, eingereicht vom Gerechtshof Arnhem Leeuwarden (Berufungsgericht Arnhem-Leeuwarden, Niederlande) mit Entscheidung vom 23. Mai 2017, beim Gerichtshof eingegangen am 29. Mai 2017, in dem Verfahren

Levola Hengelo BV

gegen

\section{Smilde Foods BV}

erlässt DER GERICHTSHOF (Große Kammer) unter Mitwirkung (...) aufgrund des schriftlichen Verfahrens und auf die mündliche Verhandlung vom 4. Juni 2018, unter Berücksichtigung der Erklärungen (...) nach Anhörung der Schlussanträge des Generalanwalts in der Sitzung vom 25. Juli 2018 folgendes

\section{Urteil}

[1] Das Vorabentscheidungsersuchen betrifft die Auslegung des Begriffs „Werk“ iSd Richtlinie 2001/29/EG des Europäischen Parlaments und des Rates vom 22.5.2001 zur Harmonisierung bestimmter Aspekte des Urheberrechts und der verwandten Schutzrechte in der Informationsgesellschaft (ABI. 2001, L 167, S. 10).

[2] Es ergeht im Rahmen eines Rechtsstreits zwischen Levola Hengelo BV (im Folgenden: Levola) und der Smilde Foods BV (im Folgenden: Smilde) wegen des angeblichen Verstoßes von Smilde gegen Rechte des geistigen Eigentums von Levola in Bezug auf den Geschmack eines Lebensmittels.

\section{Rechtlicher Rahmen}

Völkerrecht

[3] Art. 1 der Berner Übereinkunft zum Schutz von Werken der Literatur und Kunst (Pariser Fassung vom 24.7.1971) in ihrer am 28.9.1979 geänderten Fassung (im Folgenden: Berner Übereinkunft) lautet:

„Die Länder, auf die diese Übereinkunft Anwendung findet, bilden einen Verband zum Schutz der Rechte der Urheber an ihren Werken der Literatur und Kunst."
[4] In Art. 2 Abs. 1 und 2 Berner Übereinkunft heißt es:

"(1) Die Bezeichnung ,Werke der Literatur und Kunst' umfasst alle Erzeugnisse auf dem Gebiet der Literatur, Wissenschaft und Kunst, ohne Rücksicht auf die Art und Form des Ausdrucks, wie: Bücher, Broschüren und andere Schriftwerke; Vorträge, Ansprachen, Predigten und andere Werke gleicher Art; dramatische oder dramatisch-musikalische Werke; choreographische Werke und Pantomimen; musikalische Kompositionen mit oder ohne Text; Filmwerke einschließlich der Werke, die durch ein ähnliches Verfahren wie Filmwerke hervorgebracht sind; Werke der zeichnenden Kunst, der Malerei, der Baukunst, der Bildhauerei, Stiche und Lithographien; photographische Werke, denen Werke gleichgestellt sind, die durch ein der Photographie ähnliches Verfahren hervorgebracht sind; Werke der angewandten Kunst; Illustrationen, geographische Karten; Pläne, Skizzen und Darstellungen plastischer Art auf den Gebieten der Geographie, Topographie, Architektur oder Wissenschaft.

(2) Der Gesetzgebung der Verbandsländer bleibt jedoch vorbehalten, die Werke der Literatur und Kunst oder eine oder mehrere Arten davon nur zu schützen, wenn sie auf einem materiellen Trägerfestgelegt sind.“

[5] Gem. Art. 9 Abs. 1 der Berner Übereinkunft genießen die Urheber von Werken der Literatur und Kunst, die durch diese Übereinkunft geschützt sind, das ausschließliche Recht, die Vervielfältigung dieser Werke zu erlauben, gleichviel, auf welche Art und in welcher Form sie vorgenommen wird.

[6] Art. 9 des Übereinkommens über handelsbezogene Aspekte der Rechte des geistigen Eigentums in Anhang 1C des Übereinkommens zur Errichtung der Welthandelsorganisation (WTO), das am 15. April 1994 in Marrakesch unterzeichnet und durch den Beschluss 94/800/EG des Rates vom 22. Dezember 1994 über den Abschluss der Übereinkünfte im Rahmen der multilateralen Verhandlungen der Uruguay-Runde (1986-1994) im Namen der Europäischen Gemeinschaft in Bezug auf die in ihre Zuständigkeiten fallenden Bereiche (ABI. 1994, L 336, S. 1) genehmigt wurde, bestimmt:

"(1) Die Mitglieder befolgen die Artikel 1 bis 21 der Berner Übereinkunft (...) und den Anhang dazu. (...)

(2) Der urheberrechtliche Schutz erstreckt sich auf Ausdrucksformen und nicht auf Ideen, Verfahren, Arbeitsweisen oder mathematische Konzepte als solche." 
[7] Die Weltorganisation für geistiges Eigentum (WIPO) nahm am 20. Dezember 1996 in Genf den WIPO-Urheberrechtsvertrag an, der am 6. März 2002 in Kraft trat. Dieser Vertrag wurde durch den Beschluss 2000/278/EG des Rates vom 16. März 2000 (ABI. 2000, L 89, S. 6) im Namen der Europäischen Gemeinschaft genehmigt (im Folgenden: WIPO-Urheberrechtsvertrag). Art. 1 Abs. 4 dieses Vertrags sieht vor:

"Die Vertragsparteien kommen den Artikeln 1 bis 21 und dem Anhang der Berner Übereinkunft nach."

[8] Art. 2 des WIPO-Urheberrechtsvertrags bestimmt:

„Der Urheberrechtsschutz erstreckt sich auf Ausdrucksformen und nicht auf Gedanken, Verfahren, Methoden oder mathematische Konzepte als solche."

Unionsrecht

Richtlinie 2001/29

[9] Die Art. 1 bis 4 der Richtlinie 2001/29 enthalten folgende Bestimmungen:

\section{„Artikel 1}

Anwendungsbereich

(1) Gegenstand dieser Richtlinie ist der rechtliche Schutz des Urheberrechts und der verwandten Schutzrechte im Rahmen des Binnenmarkts, insbesondere in Bezug auf die Informationsgesellschaft.

(2) Außer in den in Artikel 11 genannten Fällen lässt diese Richtlinie die bestehenden [unions]rechtlichen Bestimmungen über folgende Bereiche unberührt und beeinträchtigt sie in keiner Weise:

a) über den rechtlichen Schutz von Computerprogrammen;

b) über das Vermietrecht, das Verleihrecht und bestimmte dem Urheberrecht verwandte Schutzrechte im Bereich des geistigen Eigentums;

c) über das Urheberrecht und die verwandten Schutzrechte im Bereich des Satellitenrundfunks und der Kabelweiterverbreitung;

d) über die Dauer des Schutzes des Urheberrechts und bestimmter verwandter Schutzrechte;

e) über den rechtlichen Schutz von Datenbanken. (...)
Die Mitgliedstaaten sehen für folgende Personen das ausschließliche Recht vor, die unmittelbare oder mittelbare, vorübergehende oder dauerhafte Vervielfältigung auf jede Art und Weise und in jeder Form ganz oder teilweise zu erlauben oder zu verbieten:

a) für die Urheber in Bezug auf ihre Werke, (...)

\section{Artikel 3}

Recht der öffentlichen Wiedergabe von Werken und Recht der öffentlichen Zugänglichmachung sonstiger Schutzgegenstände

(1) Die Mitgliedstaaten sehen vor, dass den Urhebern das ausschließliche Recht zusteht, die drahtgebundene oder drahtlose öffentliche Wiedergabe ihrer Werke einschließlich der öffentlichen Zugänglichmachung der Werke in der Weise, dass sie Mitgliedern der Öffentlichkeit von Orten und zu Zeiten ihrer Wahl zugänglich sind, zu erlauben oder zu verbieten. (...)

Artikel 4

Verbreitungsrecht

(1) Die Mitgliedstaaten sehen vor, dass den Urhebern in Bezug auf das Original ihrer Werke oder auf Vervielfältigungsstücke davon das ausschließliche Recht zusteht, die Verbreitung an die Öffentlichkeit in beliebiger Form durch Verkauf oder auf sonstige Weise zu erlauben oder zu verbieten. (...)"

[10] Art. 5 der Richtlinie 2001/29 nennt eine Reihe von Ausnahmen und Beschränkungen der ausschließlichen Rechte, die den Urhebern in den Art. 2 bis 4 dieser Richtlinie an ihren Werken eingeräumt werden.

Verfahrensordnung des Gerichtshofs

[11] Art. 94 der Verfahrensordnung des Gerichtshofs bestimmt:

„Das Vorabentscheidungsersuchen muss außer den dem Gerichtshof zur Vorabentscheidung vorgelegten Fragen enthalten:

a) eine kurze Darstellung des Streitgegenstands und des maßgeblichen Sachverhalts, wie er vom vorlegenden Gericht festgestellt worden ist, oder zumindest eine Darstellung der tatsächlichen Umstände, auf denen die Fragen beruhen;

b) den Wortlaut der möglicherweise auf den Fall anwendbaren nationalen Vorschriften und gegebenenfalls die einschlägige nationale Rechtsprechung;

Artikel 2

Vervielfältigungsrecht 
c) eine Darstellung der Gründe, aus denen das vorlegende Gericht Zweifel bezüglich der Auslegung oder der Gültigkeit bestimmter Vorschriften des Unionsrechts hat, und den Zusammenhang, den es zwischen diesen Vorschriften und dem auf den Ausgangsrechtsstreit anwendbaren nationalen Recht herstellt."

\section{Niederländisches Recht}

[12] Art. 1 der Auteurswet (Urheberrechtsgesetz) bestimmt:

„Das Urheberrecht ist vorbehaltlich der gesetzlichen Beschränkungen das ausschließliche Recht des Urhebers eines Werks der Literatur, der Wissenschaft oder der Kunst oder seines Rechtsnachfolgers, dieses Werk zu veröffentlichen oder zu vervielfältigen."

[13] Art. 10 Abs. 1 der Auteurswet lautet:

„Werke der Literatur, Wissenschaft oder Kunst im Sinne dieses Gesetzes sind:

1) Bücher, Broschüren, Zeitungen, Zeitschriften und alle anderen Schriftwerke,

2) dramatische und dramatisch-musikalische Werke,

3) Reden,

4) choreographische und pantomimische Werke,

5) musikalische Werke mit und ohne Text,

6) Zeichnungen, Gemälde, Bauwerke, Skulpturen, Lithografien, Gravuren und andere Arbeiten auf Blech,

7) Landkarten,

8) Entwürfe, Skizzen und plastische Darstellungen mit Bezug auf die Ingenieurwissenschaften, die Geografie, die Topografie oder andere Wissenschaften,

9) Lichtbildwerke,

10) Filmwerke,

11) Werke der angewandten Kunst sowie gewerbliche Muster und Modelle,

12) Computerprogramme und das Entwurfsmaterial sowie generell jedes Erzeugnis auf dem Gebiet der Literatur, Wissenschaft oder Kunst, unabhängig davon, auf welche Weise oder in welcher Form es zum Ausdruck gebracht wird."

Ausgangsverfahren und Vorlagefragen
[14] Der "Heksenkaas" oder "Heks'nkaas" (im Folgenden: Heksenkaas) ist ein im Jahr 2007 von einem niederländischen Gemüse- und Frischproduktehändler kreierter Streichkäse mit Crème fraîche und Kräutern. Aufgrund eines im Jahr 2011 geschlossenen Vertrags wurden die Rechte des geistigen Eigentums an diesem Erzeugnis von dessen Schöpfer gegen eine an den mit diesem Erzeugnis erzielten Umsatz geknüpfte Vergütung an Levola übertragen.

[15] Am 10. Juli 2012 wurde für das Verfahren zur Herstellung von Heksenkaas ein Patent erteilt.

[16] Seit Januar 2014 stellt Smilde ein Erzeugnis mit der Bezeichnung „Witte Wievenkaas" für eine Supermarktkette in den Niederlanden her.

[17] In der Erwägung, dass die Herstellung und der Verkauf von Witte Wievenkaas ihre Urheberrechte am "Geschmack" des Heksenkaas verletze, erhob Levola bei der Rechtbank Gelderland (Gericht Gelderland, Niederlande) Klage gegen Smilde.

[18] Da nämlich ihrer Auffassung nach das Urheberrecht an einem Geschmack auf den "gesamten durch Verzehr eines Lebensmittels hervorgerufenen Eindruck auf die Geschmackssinne einschließlich des mit dem Tastsinn wahrgenommenen Gefühls" abstelle, beantragte Levola bei der Rechtbank Gelderland (Gericht Gelderland), festzustellen, dass erstens der Geschmack von Heksenkaas eine eigene geistige Schöpfung von dessen Hersteller sei und mithin als Werk im Sinne von Art. 1 der Auteurswet urheberrechtlichen Schutz genieße und dass zweitens der Geschmack des von Smilde hergestellten Erzeugnisses eine Vervielfältigung dieses Werks darstelle. Sie beantragte ferner, Smilde aufzugeben, jegliche Verletzung ihres Urheberrechts, einschließlich der Herstellung, des Ankaufs, des Verkaufs oder einer anderen Vermarktung des als "Witte Wievenkaas" bezeichneten Produkts, abzustellen

[19] Mit Urteil vom 10. Juni 2015 befand die Rechtbank Gelderland (Gericht Gelderland), dass, ohne dass es einer Entscheidung über die Frage bedürfe, ob der Geschmack des Heksenkaas schutzfähig im Sinne des Urheberrechts sei, das Klagebegehren von Levola jedenfalls abzuweisen sei, da diese nicht angegeben habe, welche Bestandteile oder Kombination von Bestandteilen des Geschmacks des Heksenkaas diesem einen eigenen, durch Originalität geprägten Charakter und einen persönlichen Stempel verliehen.

[20] Levola legte gegen dieses Urteil Rechtsmittel beim vorlegenden Gericht ein.

[21] Nach dessen Ansicht stellt sich im Ausgangsrechtsstreit die zentrale Frage, ob der Geschmack eines Lebensmittels urheberrechtlich geschützt werden kann. Die Parteien des bei ihm anhängigen Rechtsstreits verträten hierzu entgegengesetzte Standpunkte. 
[22] Nach Auffassung von Levola kann der Geschmack eines Lebensmittels als urheberrechtlich geschütztes Werk der Literatur, der Wissenschaft oder der Kunst eingestuft werden. Levola stützt sich dabei entsprechend $u$. a. auf das Urteil des Hoge Raad der Nederlanden (Oberster Gerichtshof der Niederlande) vom 16.6.2006,

Lancôme (ECLI:NL:HR:2006:AU8940), in dem dieses Gericht grundsätzlich die Möglichkeit anerkannt habe, ein Urheberrecht am Geruch eines Parfums zuzuerkennen.

[23] Dagegen trägt Smilde vor, der Schutz eines Geschmacks sei nicht mit dem System des Urheberrechts vereinbar, das nur Schöpfungen erfasse, die optisch oder akustisch wahrgenommen werden könnten. Darüber hinaus stünden die Verderblichkeit eines Lebensmittels und der subjektive Charakter der Geschmackserfahrung einer Einordnung des Geschmacks eines Lebensmittels als urheberrechtlich geschütztes Werk entgegen. Außerdem seien die Ausschließlichkeitsrechte des Urhebers eines Werks des geistigen Eigentums und die gesetzlichen Beschränkungen dieser Rechte auf Geschmäcker praktisch nicht anwendbar.

[24] Das vorlegende Gericht führt aus, die Cour de cassation (Kassationsgerichtshof, Frankreich) habe die Möglichkeit eines urheberrechtlichen Schutzes eines Geruchs u.a. in ihrem Urteil vom 10.12.2013 (ECLI:FR:CCASS:2013:CO01205) kategorisch ausgeschlossen. Die Rechtsprechung der nationalen Obergerichte in der Europäischen Union gehe somit in der - der im Ausgangsverfahren aufgeworfenen Frage entsprechenden - Frage nach dem Urheberrechtsschutz für einen Geruch auseinander.

[25] Unter diesen Umständen hat der Gerechtshof ArnhemLeeuwarden (Berufungsgericht Arnhem-Leeuwarden, Niederlande) beschlossen, das Verfahren auszusetzen und dem Gerichtshof folgende Fragen zur Vorabentscheidung vorzulegen:

1. a) Steht das Unionsrecht einem urheberrechtlichen Schutz des Geschmacks eines Lebensmittels - als eigene geistige Schöpfung des Urhebers - entgegen? Insbesondere:

b) Steht es urheberrechtlichem Schutz entgegen, dass der Begriff "Werke der Literatur und Kunst" in Art. 2 Abs. 1 der Berner Übereinkunft, die für alle Mitgliedstaaten der Union verbindlich ist, zwar „,alle Erzeugnisse auf dem Gebiet der Literatur, Wissenschaft und Kunst, ohne Rücksicht auf die Art und Form des Ausdrucks", umfasst, sich die in dieser Vorschrift genannten Beispiele aber lediglich auf Schöpfungen beziehen, die optisch und/oder akustisch wahrgenommen werden können?

c) Stehen die (mögliche) Verderblichkeit eines Lebensmittels und/oder der subjektive Charakter der Geschmackserfahrung einer Einstufung des Geschmacks eines Lebensmittels als urheberrechtlich geschütztes Werk entgegen? d) Steht das System von Ausschließlichkeitsrechten und Beschränkungen in den Art. 2 bis 5 der Richtlinie 2001/29 dem urheberrechtlichen Schutz des Geschmacks eines Lebensmittels entgegen?

\section{Sofern Frage 1. a) verneint wird:}

a) Welche Anforderungen müssen erfüllt sein, damit der Geschmack eines Lebensmittels urheberrechtlichen Schutz genießen kann?

b) Beruht der urheberrechtliche Schutz eines Geschmacks einzig und allein auf dem Geschmack als solchem oder (auch) auf der Rezeptur des Lebensmittels?

c) Wie weit reicht die Darlegungslast einer Partei, die in einem (Verletzungs-)Verfahren geltend macht, sie habe einen urheberrechtlich geschützten Geschmack eines Lebensmittels kreiert? Kann sich diese Partei damit begnügen, dem Richter das Lebensmittel im Verfahren vorzulegen, damit sich der Richter - durch Kosten und Riechen - ein eigenes Urteil darüber bildet, ob der Geschmack des Lebensmittels den Anforderungen für urheberrechtlichen Schutz genügt? Oder hat die klagende Partei (gegebenenfalls auch) eine Beschreibung der kreativen Entscheidungen bezüglich der Geschmackskomposition und/ oder Rezeptur zu geben, aufgrund deren der Geschmack als eigene geistige Schöpfung des Urhebers anzusehen ist?

d) Wie hat der Richter in einem Verletzungsverfahren festzustellen, ob der Geschmack des Lebensmittels der beklagten Partei in der Weise mit dem Geschmack des Lebensmittels der klagenden Partei übereinstimmt, dass eine Urheberrechtsverletzung vorliegt? Kommt es in diesem Zusammenhang (auch) darauf an, ob die Gesamteindrücke beider Geschmäcker übereinstimmen?

\section{Zu den Vorlagefragen}

\section{Zur Zulässigkeit}

[26] Smilde hält das vorliegende Vorabentscheidungsersuchen für unzulässig, weil die Klage des Ausgangsverfahrens jedenfalls zurückzuweisen sei. Levola habe nämlich nicht vorgetragen, welche Merkmale des Heksenkaas diesen zu einer geistigen Schöpfung ihres Urhebers machten.

[27] Insoweit ist darauf hinzuweisen, dass es ausschließlich Sache der mit dem Rechtsstreit befassten und die Verantwortung für die abschließende richterliche Entscheidung tragenden nationalen Gerichte ist, unter Berücksichtigung der jeweiligen Einzelheiten des Rechtsstreits sowohl die Notwendigkeit einer Vorabentscheidung für die abschließende Entscheidung als auch die Erheblichkeit der dem Gerichtshof vorzulegenden Fragen zu beurteilen. Daher ist der Gerichtshof grundsätzlich gehalten, über ihm vorgelegte Fragen zu befinden, wenn 
diese die Auslegung des Unionsrechts betreffen (Urteile vom 10.3.2009, Hartlauer, C-169/07, EU:C:2009:141, Rn. 24, und vom 1.7.2010, Sbarigia, C-393/08, EU:C:2010:388, Rn. 19).

[28] Nach ständiger Rechtsprechung spricht nämlich eine Vermutung für die Entscheidungserheblichkeit der Vorlagefragen des nationalen Gerichts, die es zur Auslegung des Unionsrechts in dem rechtlichen und sachlichen Rahmen stellt, den es in eigener Verantwortung festgelegt und dessen Richtigkeit der Gerichtshof nicht zu prüfen hat. Der Gerichtshof darf die Entscheidung über ein Ersuchen eines nationalen Gerichts nur dann verweigern, wenn die erbetene Auslegung des Unionsrechts offensichtlich in keinem Zusammenhang mit der Realität oder dem Gegenstand des Ausgangsrechtsstreits steht, wenn das Problem hypothetischer Natur ist oder er nicht über die tatsächlichen oder rechtlichen Angaben verfügt, die für eine zweckdienliche Beantwortung der ihm vorgelegten Fragen erforderlich sind (Urteile vom 24.6.2008, Commune de Mesquer, C-188/07, EU:C:2008:359, Rn.30 und die dort angeführte Rechtsprechung, sowie vom 21.5.2015, Verder LabTec, C-657/13, EU:C:2015:331, Rn. 29).

[29] Im Hinblick auf die vom vorlegenden Gericht gemachten Angaben kann aber nicht festgestellt werden, dass die vorgelegten Fragen in keinem Zusammenhang mit der Realität oder dem Gegenstand des Ausgangsrechtsstreits stünden oder sich auf ein Problem hypothetischer Natur bezögen. Der bloße Umstand, dass das erstinstanzliche Gericht, dessen Entscheidung vor dem vorlegenden Gericht angefochten wird, im Unterschied zu diesem der Auffassung gewesen ist, es könne über den bei ihm anhängigen Rechtsstreit urteilen, ohne die Vorfrage zu entscheiden, ob der Geschmack eines Lebensmittels urheberrechtlichen Schutz genießen kann, führt zu keinem anderen Schluss.

[30] Außerdem ist festzustellen, dass das vorlegende Gericht dem Gerichtshof gemäß Art. 94 der Verfahrensordnung die tatsächlichen oder rechtlichen Gesichtspunkte vorgelegt hat, die für eine Beantwortung der vorgelegten Fragen erforderlich sind.

[31] Die vorgelegten Fragen sind daher zulässig.

\section{Zur ersten Frage}

[32] Mit seiner ersten Frage möchte das vorlegende Gericht wissen, ob die Richtlinie 2001/29 dahin auszulegen ist, dass sie dem entgegensteht, dass der Geschmack eines Lebensmittels durch das Urheberrecht gemäß dieser Richtlinie geschützt ist und dass nationale Rechtsvorschriften dahin ausgelegt werden, dass sie einem solchen Geschmack urheberrechtlichen Schutz gewähren.

[33] Insoweit bestimmt die Richtlinie 2001/29 in ihren Art. 2 bis 4, dass die Mitgliedstaaten ausschließliche Rechte für die Urheber in Bezug auf ihre "Werke" vorsehen. Art. 5 der Richt- linie nennt eine Reihe von Ausnahmen und Beschränkungen dieser Rechte. Die Richtlinie verweist für die Ermittlung des Sinnes und der Tragweite des Begriffs "Werk" nicht ausdrücklich auf das Recht der Mitgliedstaaten. Im Hinblick auf die Erfordernisse sowohl der einheitlichen Anwendung des Unionsrechts als auch des Gleichheitssatzes muss dieser Begriff daher in der Regel in der gesamten Union eine autonome und einheitliche Auslegung erhalten (vgl. in diesem Sinne Urteile vom 16.7.2009, Infopaq International, C-5/08, EU:C:2009:465, Rn. 27 und 28, sowie vom 3.9.2014, Deckmyn und Vrijheidsfonds, C-201/13, EU:C:2014:2132, Rn. 14 und 15).

[34] Der Geschmack eines Lebensmittels kann folglich nur dann durch das Urheberrecht gem. der Richtlinie 2001/29 geschützt sein, wenn ein solcher Geschmack als „Werk“ im Sinne dieser Richtlinie eingestuft werden kann (vgl. entsprechend Urteil vom 16.7.2009, Infopaq International, C-5/08, EU:C:2009:465, Rn. 29 und die dort angeführte Rechtsprechung).

[35] Für eine Einstufung eines Objekts als „Werk“ im Sinne der Richtlinie 2001/29 müssen insoweit zwei kumulative Voraussetzungen erfüllt sein.

[36] Zum einen muss es sich bei dem betreffenden Objekt um ein Original in dem Sinne handeln, dass es eine eigene geistige Schöpfung seines Urhebers darstellt (Urteil vom 4.10.2011, Football Association Premier League u.a., C-403/08 und C-429/08, EU:C:2011:631, Rn. 97 sowie die dort angeführte Rechtsprechung).

[37] Zum anderen ist die Einstufung als "Werk" im Sinne der Richtlinie 2001/29 Elementen vorbehalten, die eine solche geistige Schöpfung zum Ausdruck bringen (vgl. u.a. sinngemäß Urteile vom 16. Juli 2009, Infopaq International, C-5/08, EU:C:2009:465, Rn. 39, sowie vom 4.10.2011, Football Association Premier League u.a., C-403/08 und C-429/08, EU:C:2011:631, Rn. 159).

[38] Insoweit ist darauf hinzuweisen, dass sich die Union, obwohl sie nicht Vertragspartei der Berner Übereinkunft ist, nach Art. 1 Abs. 4 des WIPO-

Urheberrechtsvertrags, dem sie beigetreten ist und der mit der Richtlinie 2001/29 umgesetzt werden soll, dennoch an die Art. 1 bis 21 der Berner Übereinkunft halten muss (vgl. in diesem Sinne Urteile vom 9.2.2012, Luksan, C-277/10, EU:C:2012:65, Rn. 59 und die dort angeführte Rechtsprechung, sowie vom 26.4.2012, DR und TV2 Danmark, C-510/10, EU:C:2012:244, Rn. 29).

[39] Nach Art. 2 Abs. 1 der Berner Übereinkunft umfassen die Werke der Literatur und Kunst aber alle Erzeugnisse auf dem Gebiet der Literatur, Wissenschaft und Kunst, ohne Rücksicht auf die Art und die Form des Ausdrucks. Ferner erstreckt sich gem. Art. 2 des WIPO-Urheberrechtsvertrags und Art. 9 Abs. 2 des in Rn. 6 des vorliegenden Urteils erwähnten Übereinkommens 
über handelsbezogene Aspekte der Rechte des geistigen Eigentums, das ebenfalls Teil der Rechtsordnung der Union ist (vgl. in diesem Sinne Urteil vom 15.3.2012, SCF, C-135/10, EU:C:2012:140, Rn. 39 und 40), der urheberrechtliche Schutz auf Ausdrucksformen und nicht auf Ideen, Verfahren, Arbeitsweisen oder mathematische Konzepte als solche (vgl. in diesem Sinne Urteil vom 2.5.2012, SAS Institute, C-406/10, EU:C:2012:259, Rn. 33).

[40] Der Begriff „Werk“, auf den die Richtlinie 2001/29 abzielt, impliziert daher notwendigerweise eine Ausdrucksform des urheberrechtlichen Schutzobjekts, die es mit hinreichender Genauigkeit und Objektivität identifizierbar werden lässt, auch wenn diese Ausdrucksform nicht notwendigerweise dauerhaft sein sollte.

[41] Zum einen müssen nämlich die Behörden, die mit dem Schutz der dem Urheberrecht innewohnenden Ausschließlichkeitsrechte betraut sind, die so geschützten Objekte klar und genau erkennen können. Dasselbe gilt für Privatpersonen, insbesondere Wirtschaftsteilnehmer, die mit Klarheit und Genauigkeit die Objekte identifizieren können müssen, die zugunsten von Dritten, insbesondere Wettbewerbern, geschützt sind. Zum anderen impliziert das Erfordernis des Ausschlusses jedes - der Rechtssicherheit schädlichen - subjektiven Elements bei der Identifizierung des geschützten Objekts, dass dieses Gegenstand eines präzisen und objektiven Ausdrucks sein kann.

[42] An der Möglichkeit einer präzisen und objektiven Identifizierung fehlt es aber im Fall des Geschmacks eines Lebensmittels. Im Unterschied zu beispielsweise einem literarischen, bildnerischen, filmischen oder musikalischen Werk, das eine präzise und objektive Ausdrucksform darstellt, beruht die Identifizierung des Geschmacks eines Lebensmittels nämlich im Wesentlichen auf Geschmacksempfindungen und -erfahrungen, die subjektiv und veränderlich sind, da sie u.a. von Faktoren, die mit der Person verbunden sind, die das betreffende Erzeugnis kostet, wie beispielsweise deren Alter, Ernährungsvorlieben und Konsumgewohnheiten, sowie von der Umwelt oder dem Kontext, in dem dieses Erzeugnis gekostet wird, abhängen.

[43] Zudem ist beim gegenwärtigen Stand der Wissenschaft eine genaue und objektive Identifizierung des Geschmacks eines Lebensmittels, die es erlaubt, ihn vom Geschmack anderer gleichartiger Erzeugnisse zu unterscheiden, mit technischen Mitteln nicht möglich.

[44] Nach alledem ist daher festzustellen, dass der Geschmack eines Lebensmittels nicht als „Werk" im Sinne der Richtlinie 2001/29 einzustufen ist.

[45] In Anbetracht des in Rn. 33 des vorliegenden Urteils angeführten Erfordernisses einer einheitlichen Auslegung des Begriffs "Werk" in der Union ist zudem festzustellen, dass die Richtlinie 2001/29 es verwehrt, nationale Rechtsvorschriften dahin auszulegen, dass sie dem Geschmack eines Lebensmittels urheberrechtlichen Schutz gewähren.
[46] Folglich ist auf die erste Frage zu antworten, dass die Richtlinie 2001/29 dahin auszulegen ist, dass sie dem entgegensteht, dass der Geschmack eines Lebensmittels durch das Urheberrecht gemäß dieser Richtlinie geschützt ist und dass nationale Rechtsvorschriften dahin ausgelegt werden, dass sie einem solchen Geschmack urheberrechtlichen Schutz gewähren.

\section{Zur zweiten Frage}

[47] In Anbetracht der Antwort auf die erste Frage ist die zweite Frage nicht zu beantworten.

Kosten

[48] Für die Parteien des Ausgangsverfahrens ist das Verfahren ein Zwischenstreit in dem beim vorlegenden Gericht anhängigen Rechtsstreit; die Kostenentscheidung ist daher Sache dieses Gerichts. Die Auslagen anderer Beteiligter für die Abgabe von Erklärungen vor dem Gerichtshof sind nicht erstattungsfähig.

Aus diesen Gründen hat der Gerichtshof (Große Kammer) für Recht erkannt:

Die Richtlinie 2001/29/EG des Europäischen Parlaments und des Rates vom 22.5. 2001 zur Harmonisierung bestimmter Aspekte des Urheberrechts und der verwandten Schutzrechte in der Informationsgesellschaft ist dahin auszulegen, dass sie dem entgegensteht, dass der Geschmack eines Lebensmittels durch das Urheberrecht gemäß dieser Richtlinie geschützt ist und dass nationale Rechtsvorschriften dahin ausgelegt werden, dass sie einem solchen Geschmack urheberrechtlichen Schutz gewähren. 